\title{
Processing parameters influence on dynamics of vibratory drilling with adaptive control
}

\author{
Ilya I. Ivanov*, and Sergey A. Voronov \\ Bauman Moscow State Technical University, 105005 Moscow 2-ya Baumanskaya ul. 5, Russia
}

\begin{abstract}
Chip segmentation is one of necessary conditions of ensuring the deep hole drilling quality when processing hard-to-machine metals. It may be achieved through making drill bit harmonic oscillations in axial direction. Possible way to maintain such vibrations is to replace the standard drilling head with special vibratory head which includes elastic element giving the instrument a possibility to move in axial direction. Self-excitation of drill regenerative oscillations is possible if elastic element stiffness and processing parameters are chosen properly. It is advisable to complement this way of excitation by control action which is determined in feedback circuit and sustains required vibration process characteristics in wide range of processing parameters. In present paper the adaptive control algorithm of vibratory drilling process dynamics is proposed. Control action on oscillation system is proportional to drill vibrational velocity, the feedback gain is determined in adaptation circuit basing on comparison of actual peakto-peak vibrational displacement and its target value. Simulation of closedloop nonlinear system «elastic system - machining process - control system» dynamics has been performed for different values of processing parameters for cases with or without control. The simulation revealed efficiency of suggested algorithm in wide range of processing parameters. Joined influence of processing parameters and target peak-to-peak displacement values on chip breakage conditions is studied. Recommendations for choice of processing parameters and control parameters values are developed. Influence of control action magnitude limit on control system possibilities to achieve control target is analyzed.
\end{abstract}

\section{Introduction}

During drilling deep holes in metal workpieces flow chip is usually produced [1]. It can be wound on an instrument causing difficulties of chip removal, possible seizing and breakage of the tool, damage of processed workpiece surface. This problem is especially strong while cutting hard-to-machine metals and alloys. Because of the listed reasons chip segmentation is necessary during treatment process. If chip is being broken drilling can be performed continuously without standstills, process productivity and quality do increase.

One of ways to ensure chip segmentation is making drill bit to oscillate in axial direction. If cutting edges periodically leave cutting zone, segmented chip is produced, which is easy

\footnotetext{
* Corresponding author: ivanovilig@gmail.com
} 
to remove from the hole. The requirements on the vibration amplitude and frequency for chip breakage are formulated in [2]. It was shown that minimal peak-to-peak (PTP) value of vibration displacement necessary for chip segmentation should be not less than feed per cutting edge.

At present different designs of devices for imparting fixed harmonic law to the tool displacements have been developed and tried: with hydraulic [3], kinematic [4,5] and other excitation types. High reliability of chip breakage and absence of time delay between process start and segmentation initiation are the advantages of these devices. The necessity of machine tool design changes as well as requirement for supply of a large amount of energy on the rotating spindle are the drawbacks.

Besides the devices of the listed types there exist special vibratory heads [6] using main motion energy for maintenance the required axial drill oscillations. The vibratory heads (fig. 1) include elastic element which transduces axial force. Because of flexibility introduced by the elastic element the tool and the movable (in axial direction) part of the head can oscillate relative to the unmovable part of the head and the spindle. Herewith the drill axial vibrations are being self-excited by regenerative mechanism [7] due to main motion energy. The review of researches, devoted to different models and analysis methods of self-excited axial vibrations for vibratory drilling, is presented in [8]. The study of vibrations of the system, including flexible drill and vibratory head, is performed in [9].

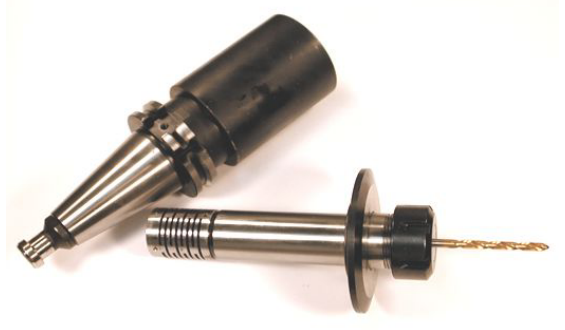

Fig. 1. Photography of the head with introduced elastic element of slotted type.

Researches [10,11] showed that amplitude and frequency of the steady-state self-excited vibrations are determined by vibratory head dynamic properties, specified cutting conditions, workpiece material properties and drill bit geometric characteristics. Under specified conditions self-excited tool vibrations characteristics can be either insufficient or excessive for ensuring chip segmentation. Fine adjustment of dynamic vibratory head properties and cutting conditions is required. If there is lack of exact information about system damping, change of cutting conditions and tool parameters because of cutting edges wear, the reliability of self-excited vibrations maintenance is not high.

The possible way to correct the described drawback of vibratory heads is to complement the self-excitation mechanism by external control action, built in feedback circuit. The control system should supply energy to the oscillatory system, if vibrations amplitudes are not enough for chip segmentation, and should decrease the amplitudes in the opposite case. The vibratory drilling dynamics control algorithm meeting this requirement was suggested in study [12]. However, study [13] shows that unstable unlimited oscillations are possible if this algorithm is used. Alternative control algorithms were suggested in research [14], but their application area is bounded by the case when electro-mechanical transformation is purely linear. So the practical implementation field of these algorithms is significantly limited. Thus the problem of development of vibratory drilling control system is actual.

In the present paper the new vibratory drilling dynamics control strategy with feedback gain adaptation based on the PTP oscillation displacement is described and studied. The mathematical model equations are presented in section 2 . The control strategy and integral characteristics of vibratory chip segmentation process are described in section 3 . The results 
of system dynamics simulation for the two cases, without and with control, and study of dependency of vibratory chip segmentation process quality on cutting conditions and control system parameters are presented in section 4 . Section 5 contains conclusions.

\section{Mathematical model}

The computational model of controlled vibratory drilling process dynamics with kinematic type of excitation is shown in the fig. 2. This type of actuation is implemented by means of giving some displacement $x_{a}$ to the vibratory head elastic element fastening. This displacement can be imparted by built-in piezo actuator, for example.

Controlled vibratory drilling dynamics equations have been derived by authors in [13] and here are adduced in non-dimensional form without mathematical treatments:

$$
\begin{gathered}
\frac{1}{(2 \pi p)^{2}} \ddot{q}+\frac{\zeta}{\pi p} \dot{q}+q=P_{c}+q_{0} \\
\eta(\tau)=(\Lambda(\tau-1)+1-q(\tau)) H(\Lambda(\tau-1)+1-q(\tau)) \\
P_{c}=k_{c} \eta^{r} \\
\Lambda(\tau)=\Lambda(\tau-1)+1-\eta(\tau)
\end{gathered}
$$

where $q$ - the unitless axial tool coordinate, which is measured by feed parts; $p$ - the ratio of the vibratory head eigenfrequency to the tool cutting edges pass frequency; $\zeta$ - the unitless damping coefficient; $P_{c}$ - the unitless cutting force, which is measured by parts of multiplication of elastic element stiffness by feed per cutting edge; $q_{0}$ - the unitless kinematic excitation, which is measured by feed parts; $\eta$ - the unitless cut-off thickness, which is measured by feed parts; $\tau$ - the unitless time, which is measured by parts of cutting edges pass period; $\Lambda$ - the unitless axial coordinate of the surface being processed; $k_{c}, r$-empirical constants, depending on workpiece material properties, diameter and cutting part geometrical form of the drill. In further computations $r$ is equal 0.7 .

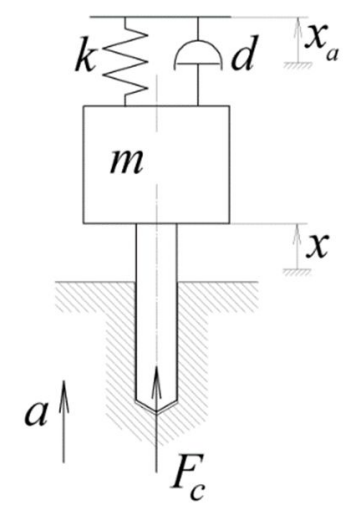

Fig. 2. The computational model of vibratory drilling with control for case of kinematic excitation.

The described system of equations (1) - (4) is nonlinear with time delay introduced in equations (2), (4). In the current study the unknowns $\left\{q, \eta, P_{c}, \Lambda\right\}$ are computed numerically on discrete time mesh with a constant step $\Delta \tau: \tau_{j}=j \Delta \tau, j=0,1,2, \ldots$ The method of successive approximations with iterative improvement of the state vector at the end of the step is applied at every $j$-th time step. 


\section{Control algorithm. Vibratory chip segmentation process quality characteristics}

Let us take the following control law:

$$
q_{0}=k_{y} b \dot{q}
$$

where $k_{y}$ - the control signal amplification factor, $b$ - the feedback gain which is set by the controller. As the usage of a digital controller is assumed, $b$ may be equal to a discrete series values. For example, in the case of 10 -bit digital-analog converter $-(0 \ldots 1023)$ or ($512 \ldots 511)$. The amplification factor is assumed to be equal to $k_{y}=5 \cdot 10^{-5}$ here and further if other value is not specified.

We assume the following adaptation algorithm of feedback gain in the research:

$$
\dot{b}=-c_{1}\left(\frac{A}{A_{0}}-1\right)-c_{2} \frac{\dot{A}}{A_{0}}
$$

where $A$ - the current PTP vibration displacement value, $A_{0}$ - the target PTP displacement, $c_{1}, c_{2}-$ the adaptation parameters.

This algorithm results in feedback gain decrease, if the PTP vibration displacement value A is higher than its target value $A_{0}$, and in increase, if the PTP displacement is less than target value.

Thus the system of equations (1) - (6) is modelled jointly. The single simulation results are the time dependencies of the unitless displacement and the unitless cutting force. The results of multi-variant modelling are the dependencies of some vibratory steady-state integral characteristics on system model parameters. It is assumed $c_{1}=c_{2}=500$ in the following calculations.

Besides the PTP oscillation displacement value $A$, the cutting continuity index is a such integral characteristic:

$$
\psi=\frac{1}{T_{A}} \int_{\tau_{f}-T_{A}}^{\tau_{f}} H\left(P_{C}(\tau)\right) d \tau
$$

where $\tau_{f}$ - the unitless simulation time; $T_{A}$ - the unitless time interval under consideration. Let us note that satisfying of $\psi<1$ condition is necessary and sufficient for chip segmentation. $T_{A}$ is assumed to be equal $1 / 5$ of $\tau_{f}$ in simulation results analysis.

\section{Simulation results comparison for the cases without and with control}

Fig. 3 presents the time dependencies of displacement and thrust cutting force computed for the cases with and without control. The control target PTP displacement values $A_{0}=1,5$. It is clear from fig. 3 that without control the vibrations decrease and the cutting operation mode with continuous chip is stabilized. In the case with control we see vibrations excitation and the vibratory operation mode with the specified PTP displacement value is maintained. Herewith cutting force periodically reach zero value that means chip breakage. 

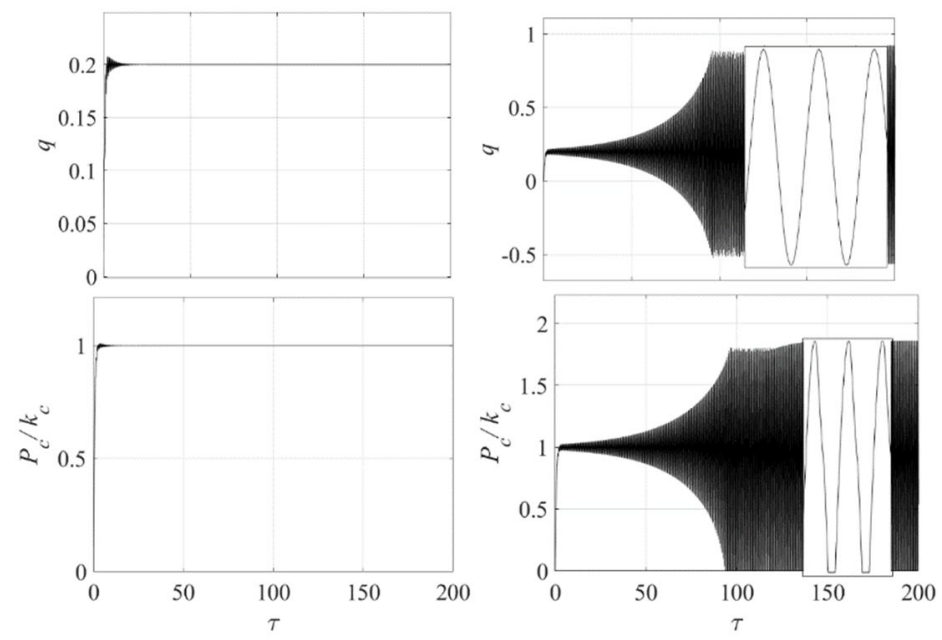

Fig. 3. Time histories of displacements (top) and thrust cutting forces (bottom) for the cases without (left) and with (right) control, $p=1.5, k_{c}=0.2$.

Further we study joined influence of the system parameters $p$ and $k_{c}$ on vibratory drilling process dynamics. Maps of dependencies of steady-state PTP displacement values on these parameters are shown in the fig. $4 a, 4 b$ for the cases without control and with control correspondingly. Analysis of presented maps lets us conclude that introducing of control leads to smoothing of steady-state PTP displacement value at a wide range of cutting conditions. The white zone in the fig. $4 b$ is caused by insufficiency of the amplification factor $k_{y}$ for ensuring the oscillations excitation for the fixed combination of cutting conditions. The map of dependency of the PTP vibration displacement value on the system parameters for the case of doubled amplification factor is shown in the fig. $4 c$. Here the area without oscillations decreases, but the lowering of control quality is possible for the high values of $k_{c}$.

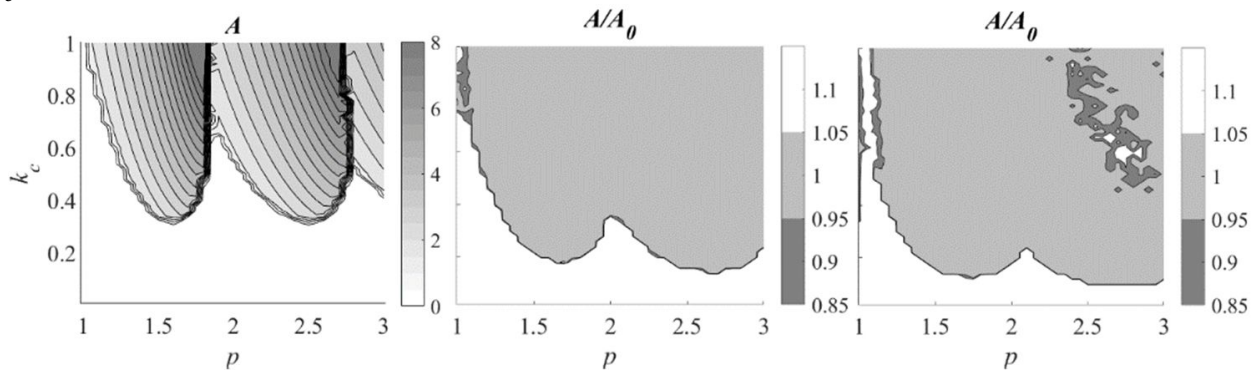

Fig.4. Maps of steady-state PTP vibration dependencies on parameters $p, k_{c}$ for cases: without control (left), control with nominal amplification factor $k_{y}$ (middle) and control with doubled amplification factor (right), $A_{0}=1.5$.

Thus the developed control system can ensure the specified PTP displacement value in a wide range of cutting conditions if the right value of amplification factor has been chosen. The problem of peak-to-peal displacement target value $A_{0}$ choice is of practical importance. Maps of the dependencies of the steady-state PTP vibration displacement and cutting continuity index on vibratory processing parameters $p, A_{0}$ are shown in the fig. $5 a, 5 b$ correspondingly. In a wide range of parameter $p$ values the control system ensures the control target almost for every possible value of a target PTP displacement $A_{0}$. We can see this from vertical character of areas borders in the fig. $5 a$. In neighbourhood of integer parameter $p$ 
control system does not achieve target PTP vibration displacement. This is because regenerative effect in those areas results in damping of oscillations, not excitation.

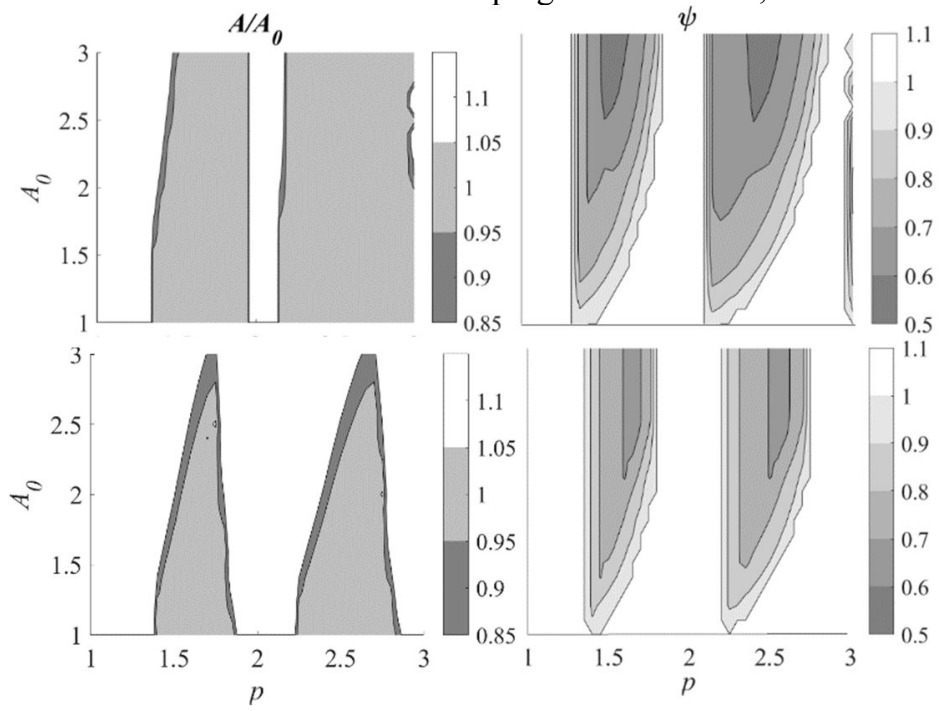

Fig. 5. Maps of dependencies of steady-state PTP displacement $A$ (left $)$ and cutting continuity index $\psi$ (right) on parameters $p, A_{0}$ for cases without (top) and with (bottom) actuator elongation limit, $k_{c}=0.3$.

Let us note that achieving the PTP vibration displacement target does not result in chip segmentation guaranteed. We can see this from comparison of fig. $5 a$ and $5 b$. Therefore, it is important to properly make the joint choice of processing parameter $p$ and target PTP displacement value $A_{0}$, basing on the condition of ensuring the required cutting continuity index (fig. $5 b$ ). If we choose value of the parameter $p$, we can set the ratio between oscillations frequency and cutting edge pass frequency, which is favourable [2] for chip breakage. In this case the control system maintains the vibratory operation conditions with specified necessary PTP displacement value and chip would be segmented.

The results, presented earlier, are computed for the case without actuator elongation limit. If we take a look at results (fig. $5 c, 5 d)$ computed with account of this limit $\left(\left|q_{a}\right| \leq 0.1 \cdot \mathrm{k}_{\mathrm{c}}\right)$, we can see that there is decrease of areas with vibration excitation and chip segmentation. This fact should be considered when projecting vibratory drilling technology.

Thus the method of chip segmentation ensuring includes the parameter $p$ and target PTP displacement $A_{0}$ choice and performing of technological operation at specified processing conditions with control system turned on.

\section{Summary}

The vibratory drilling dynamics model and adaptive control algorithm of this process are described in the paper. Basing on the mathematical model the study of closed-loop system behaviour has been performed. It was shown that implementation of control ensures the required vibratory operation mode in a wide range of cutting conditions. It is necessary to jointly choose the spindle rotation velocity and target peak-to-peak displacement value for guaranteed chip segmentation. This choice can be made basing on multi-variant simulation of closed-loop system dynamics. Integer values of ratio between elastic system eigenfrequency and cutting edges pass frequency are undesirable for chip breakage. The area of acceptable vibratory drilling cutting conditions slightly reduces if actuator elongation limit becomes lower. 
The reported study was funded by RFBR according to the research project № 18-31-00147.

\section{References}

1. E. Oezkaya, S. Mikel, D. Biermann, Prod. Eng. 12, 11 (2018)

2. V. Poduraev, Obrabotka rezaniem s vibratsiyami (Mashinostroenie, Moscow, 1970)

3. V. Popov, E. Vainshenker, M. Margulis, USSR Patent 510351 (1976)

4. G. Peigne, FR Patent 0001024 (2007)

5. J. Jallageas, J. K'nevez, M. Cherif et al., Int. J. Avd. Man. Tech. 67, 1205 (2013)

6. D. Brun-Picard, A. Gouskov, RF Patent 2212984 (2003)

7. Y. Altintas, Manufacturing Automation (Cambridge University Press, 2012)

8. S. Voronov, A. Gouskov, I. Ivanov et al., Sc. and Ed. Bauman MSTU 12, 842 (2014)

9. I. Kiselev, N. Zhukov, A. Selivanov et al., Proc. Eng., 176, 50 (2017)

10. A. Gouskov, S. Voronov, S. Batzer, Dyn. Ac. Sim., ASME 68, 263 (2000)

11. S. Tichkiewitch, G. Moraru, D. Brun-Picard, A. Gouskov, CIRP Ann. 51, 311 (2002)

12. A. Gouskov, Razrabotka metodov postroeniya $i$ analiza dinamicheskih modeley tekhnologicheskih protsessov pri mekhanicheskoy obrabotke (Doctoral Diss., 1997)

13. A. Gouskov, S. Voronov, I. Ivanov et al., JVE 17, 3702 (2015)

14. A. Gouskov, S. Voronov, I. Ivanov et al., JVE 17, 4301 (2015) 